\title{
Antinociception Produced by an Ascending Spino-Supraspinal Pathway
}

\author{
Robert W. Gear ${ }^{1}$ and Jon D. Levine ${ }^{2}$ \\ ${ }^{1}$ Graduate Program in Oral Biology and ${ }^{2}$ Departments of Medicine, Anatomy, and Oral and Maxillofacial Surgery, \\ University of California, San Francisco, California 94143
}

\begin{abstract}
Studies in mice and rats have shown that antinociception produced by intrathecal (i.t.) administration of opioids can be partially inhibited by intracerebroventricular (i.c.v.) administration of naloxone. In this study we tested the hypothesis that this inhibition by i.c.v. naloxone results from antagonism of supraspinal endogenous opioid-mediated antinociception produced by the action of i.t. opioids on an ascending antinociceptive pathway. In rats lightly anesthetized with urethane/alpha-chloralose, i.t. DAMGO, i.t. lidocaine, or spinal transection at $T_{5}-T_{6}$ all attenuated the trigeminal jaw opening reflex (JOR) (i.e., were antinociceptive), an effect that was antagonized in each case by i.c.v. naloxone. These findings support the suggestion that there exists a pathway that ascends from the spinal cord to a supraspinal site that tonically inhibits antinociception mediated by supraspinal opioids. When activity in this ascending pathway is suppressed (e.g., by i.t. opioids or local anesthetics or by spinal cord transection), antinociception mediated by supraspinal opioids is disinhibited.

To determine the supraspinal site(s) at which endogenous opioid-dependent antinociception is evoked by i.t. opioids, we microinjected naloxone methiodide into several supraspinal sites. Microinjection of naloxone methiodide into nucleus accumbens but not into the rostral ventral medulla (RVM) or the periaqueductal gray matter (PAG) antagonized the suppression of the JOR produced by i.t. DAMGO or lidocaine. The possibility that this ascending pathway may represent a source of spinal input to mesolimbic circuitry involved in setting the state of sensorimotor reactivity to noxious stimuli is discussed.
\end{abstract}

[Key words: antinociception, ascending pathway, DAMGO, intrathecal, lidocaine, nucleus accumbens, opioids, periaqueductal gray, rostral ventral medulla, spinal, supraspinal]

A large body of research has implicated endogenous opioids in the modulation of pain at three principal CNS sites, the periaqueductal gray (PAG), the rostral ventral medulla (RVM), and the dorsal horn of the spinal cord. Since electrical stimulation or

\footnotetext{
Received June 27, 1994; revised Sept. 29, 1994; accepted Nov. 7, 1994.

We thank Drs. Allan Basbaum, Howard Fields, Michael Gold, Mary Heinricher, Peggy Mason, Arthur Miller, David Reichling, and Kimberly Tanner for many helpful discussions during the course of this work. This work was supported by NIH Grants T32-DE07204 and DE08973.

Correspondence should be addressed to Jon D. Levine, M.D., Ph.D., Division of Rheumatology, S-1334 (Box 0452), University of California at San Francisco, San Francisco, CA 94143-0452A.

Copyright (C) 1995 Society for Neuroscience $0270-6474 / 95 / 153154-08 \$ 05.00 / 0$
}

microinjection of morphine into more rostral sites (i.e., the PAG or RVM) increases the thresholds of spinal nociceptive reflexes, it has been suggested that this circuit functions as a descending antinociceptive control (Basbaum and Fields, 1978; Basbaum and Fields, 1984; Fields and Basbaum, 1994). Recent studies, however, provide evidence that there is also an ascending (i.e., a spino-supraspinal) antinociceptive pathway through which spinal opioids evoke antinociception mediated by endogenous opioids at a supraspinal site(s) (Fig. 1A). For example, exogenous (Holmes and Fujimoto, 1992; Miaskowski and Levine, 1992) or endogenous (Welch et al., 1992; but see Lux et al., 1988) spinal opioids produce antinociception that can be partially antagonized by intracerebroventricular (i.c.v.) naloxone. However, these observations can be explained by alternative interpretations. For example, it has been proposed that i.c.v. naloxone "activates" a descending antianalgesia system that antagonizes the antinociceptive action of i.t. opioids at the level of the spinal cord (Holmes and Fujimoto, 1992) (Fig. 1B). Therefore, to determine if spinally administered opioids can act via an ascending spino-supraspinal circuit to produce analgesia, we devised an experimental model that separates the site of reflex measurement from the spinal site of opioid administration. In the lightly anesthetized rat, we measured the effect of intrathecally administered [D- $\mathrm{Ala}^{2}, \quad N$-Me-Phe ${ }^{4}, \mathrm{Gly}^{5}$-ol]-enkephalin (DAMGO) on the amplitude of the jaw opening reflex (JOR) with or without naloxone administered into the third cerebral ventricle (i.c.v. naloxone). To further characterize the ascending pathway (i.e., whether it must be activated in order to evoke antinociccption mediated by supraspinal opioids, or if its activity must be suppressed in order to disinhibit supraspinal opioidmediated antinociception), the effects on the JOR of i.t. lidocaine or spinal transection with or without i.c.v. naloxone were compared. Finally, to determine the supraspinal site at which naloxone acts to antagonize antinociception produced by i.t. DAMGO, we studied the effect of naloxone methiodide microinjected into several supraspinal sites on the ability of i.t. DAMGO to attenuate the JOR.

Some of the results of this study have been previously reported in abstract form (Gear and Levine, 1994).

\section{Materials and Methods}

The experiments were performed on $250-450$ gm male Sprague-Dawley rats (Bantin and Kingman, Fremont, CA) that were lightly anesthetized by intraperitoneal injection of $0.9 \mathrm{gm} / \mathrm{kg}$ urethane and $45 \mathrm{mg} / \mathrm{kg}$ $\alpha$-chloralose (both from Sigma, St. Louis, MO), and $10 \mathrm{mg} / \mathrm{kg}$ methohexital (Brevital) for rapid induction of anesthesia. We chose urethane/ $\alpha$-chloralose for anesthesia because in preliminary experiments this anesthetic, unlike a single dose of pentobarbital or continuous infusion of methohexital, provided a stable JOR EMG (see below) over the time 
A

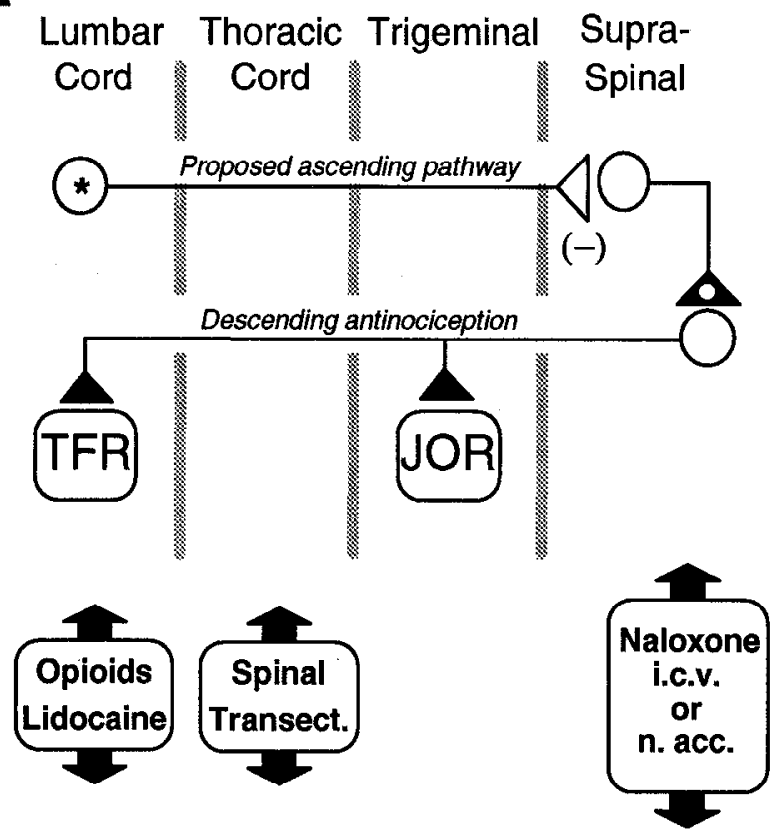

B

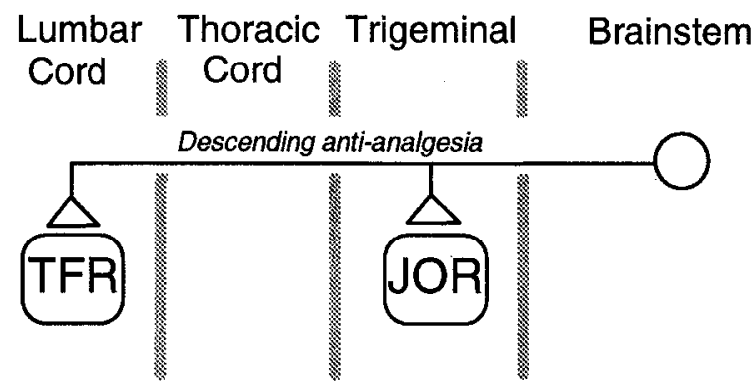

$=$ Inhibits nociceptive behavior

$=$ Inhibits nociceptive behavior (opioid)

$\triangle=$ Enhances nociceptive behavior

* = Tonically active

$(-)$ = Inhibitory synapse

TFR = Tail flick reflex JOR = Jaw opening reflex

n. acc. $=$ nuc. accumbens i.c.v = intracerebroventricular

Figure 1. A, Schematic illustration of the proposed ascending antinociceptive pathway. The asterisk indicates the tonically active ascending limb of the pathway that inhibits supraspinal opioidergic neurons (thereby enhancing nociceptive behavior) in nucleus accumbens. Suppression of activity in this ascending patliway by lumbar (i.t.) opivids or lidocaine, or thoracic spinal transection results in supraspinal opioid disinhibition which modulates spinal and trigeminal nociceptive reflexes as indicated by the solid triangles. Note that spinal analgesic agents (opioids or lidocaine), applied to the lumbar cord, act through two mechanisms hy (1) directly on the local synapses mediating the tailflick reflex $(T F R)$, and (2) suppressing the tonically active ascending pathway. Since the JOR is mediated by neuronal circuits located at a site distant from the lumbar cord, drugs applied to the lumbar cord can modulate the JOR only via an ascending pathway. Spinal transection at period (at least $3 \mathrm{hr}$ ) required to complete the experiments (data not shown). The animals were sacrificed by overdose of pentobarbital unless it was necessary to section their brains for histological purposes, in which case we sacrificed the animals by intracardiac perfusion of $4 \%$ formalin after ensuring that they were in a deep state of anesthesia with pentobarbital.

Cannulation. To administer drugs to the area of the lumbar enlargement of the spinal cord, an i.t. catheter (PE-10, $10 \mu$ l volume) was inserted $8.5 \mathrm{~cm}$ caudally into the subarachnoid space through a slit in the atlanto-occipital membrane (Yaksh and Rudy, 1976). It was not possible to determine by pharmacological/behavioral methods whether the catheters were correctly placed or whether any of the catheter placements would have resultcd in motor deficits bccause the animals do not recover from urethane/ $\alpha$-chloralose anesthesia. However, in many experiments we checked catheter placement by injecting Evans blue dye and performing a postmortem laminectomy to determine the location of the dye and the tip of the catheter. In all cases we observed that the position of the catheter varied only in its dorsoventral relationship to the cord. That is, the tip of the catheter was sometimes positioned more dorsally to the cord and sometimes it was positioned more ventrally, but the catheter never turned back on itself or perforated the dura. Also, while animals free of motor deficits would have been essential if we were measuring lumbar spinal reflexes which depend upon intact motor circuitry in the spinal cord (e.g., paw-withdrawal or tail-flick), in this study we employed the supraspinally mediated JOR.

To administer drugs to the third cerebral ventricle, an i.c.v. guide cannula (22 gaugc) was positioned to allow drug delivery via insertion of a 30 gauge injection cannula. At the conclusion of the experiments i.c.v. sites were verified by injection of Evans blue dye, equal in volume to the drug injection, after which the brains were removed, sectioned and examined for dye location. In some experiments 25 gauge guide cannulae were positioned to allow microinjections via insertion of a 33 gauge injection cannula into specific supraspinal sites. These injection sites were verified by histological examination ( $70 \mu \mathrm{m}$ sections stained with cresyl violet acetate) (see Figs. 8, 9).

Spinal transection. In experiments in which the spinal cord was transected, a laminectomy was performed after implantation of the i.c.v. cannula and the stimulating and recording electrodes for the JOR. The spinal cord was exposed, but not sectioned, by the removal of the dorsal portions of the $T_{5}$ and $T_{6}$ vertebrae. Spinal transection was performed after baseline JOR recordings and administration of i.c.v. naloxone (or vehicle) as described in "Results." These animals did not receive an i.t. catheter.

Jaw opening reflex. A bipolar stimulating electrode, fabricated from two insulated single-stranded copper wires ( $36 \mathrm{AWG}$ ), each with 0.2 $\mathrm{mm}$ of insulation removed from the tip, one tip extending $2 \mathrm{~mm}$ beyond the other, was inserted into the pulp of a mandibular incisor to a depth of $20 \mathrm{~mm}$ from the incisal edge of the tooth to the tip of the longest wire (Toda et al., 1981). Access to the pulp of the incisor was through an opening in the labial surface of the tooth starting $2 \mathrm{~mm}$ below the gingival crest and extending $4 \mathrm{~mm}$ toward the incisal edge. Dental composite resin held the electrode in place and sealed the opening in the tooth. A bipolar recording electrode, consisting of two wires of the same material as the stimulating electrode with $4 \mathrm{~mm}$ of insulation removed, was inserted into the digastric muscle ipsilateral to the implanted tooth

$\leftarrow$

the thoracic cord also modulates nociception via the ascending pathway; lowever, this can only be observed in the JOR (or other supraspinally mediated nociceptive reflexes) since reflexes mediated at the lumbar cord are disconnected from supraspinal influence. $B$, Schematic illustration of a descending antianalgesia circuit (Holmes and Fujimoto, 1992). In this model opioids or lidocaine applied to the lumbar cord would only affect lumbar spinal reflexes (e.g., the TFR) by local action. The JOR is not affected since this model does not propose an ascending pathway. When i.c.v. naloxone is administered, anti-analgesic circuits are activated in the spinal cord (open triangles), but since i.c.v. naloxone alone has no effect on nociceptive thresholds, these substances act only to antagonize the action of endogenous or exogenous spinal opioids. Since the opioids in our experiments are administered to the lumbar region of the spinal cord, the antianalgesic substances have no effect on the trigeminal JOR. Also, these antianalgesic substances are not proposed to antagonize the antinociceptive action of lidocaine. Spinal transection has no effect on the JOR in this model. 


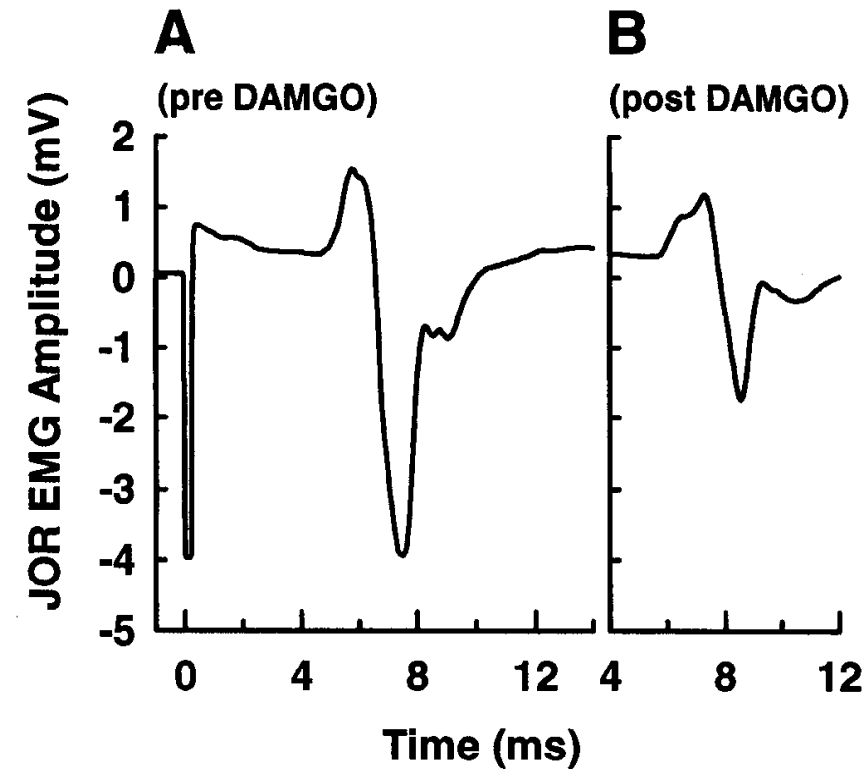

Figure 2. Examples of JOR EMG recordings. Tracings represent the average EMG in response to 12 tooth pulp stimuli. The JOR EMG response occurs with a latency of approximately $7-9 \mathrm{msec}$ after tooth pulp stimulation shown as the downward stimulus artifact at the beginning of the sweep in $A$. The peak-to-peak distance (in $\mathrm{mV}$ ) of the EMG signal was taken to be the magnitude of the EMG. $A$, A typical baseline EMG recording. Amplitude, $5.57 \mathrm{mV}$. B, Average EMG response 15 min after the administration of i.t. DAMGO $(7.5 \mu \mathrm{g})$. Amplitude, 2.91 $\mathrm{mV}$. In this example the percent decrease (i.e., "JOR suppression") is $48 \%$. Formula: $[(A-B) / A] \times 100$. Baseline values used in calculating the results of the experiments were based on three pretreatment recordings (12 stimuli each) taken $5 \mathrm{~min}$ apart.

sufficiently deep to completely submerge the uninsulated end of the wire. A 22 gauge needle was inserted in the skin ventral to the midline of the mandible and connected to the ground terminal of the amplifier. Tooth pulp was stimulated with $0.2 \mathrm{msec}$ square wave pulses at 0.33 $\mathrm{Hz}$. Stimulation voltage was set at three times the threshold voltage for evoking the JOR EMG. Twelve consecutive evoked EMG signals were averaged per recording (Fig. 2).

Antinociception was measured as the percentage decrease (mean \pm SEM) from the average amplitude of three baseline recordings taken at five minute intervals. ANOVA and either the Student-Neuman-Keuls (SNK) test or the Fisher's test (Fisher, 1949), as appropriate, were used to compare groups for significant differences $(p \leq 0.05)$.

Drugs. Lidocaine (4\% xylocaine, Astra Pharmaceutical Products, Westborough, MA) without epinephrine was used as supplied by the manufacturer. [D-Ala ${ }^{2}, \mathrm{~N}$-Me-Phe ${ }^{4}, \mathrm{Gly}^{5}$-ol]-enkephalin (DAMGO) and naloxone (Sigma, St. Louis, MO), and naloxone methiodide (Research Biochemicals International, Natick, MA), a quaternary derivative of naloxone, which has been shown to spread from the site of injection more slowly than naloxone (Schroeder et al., 1991), were dissolved in physiological saline $(0.9 \%$ ), or artificial CSF (Leviel et al., 1989). To retard rostral flow of the i.t. drug or vehicle, all animals were placed in a prone position on an inclined surface (approximately $30^{\circ}$ ) with the head higher than the tail. I.t. drug or vehicle volumes were $15 \mu$ followed by $10 \mu \mathrm{l}$ of vehicle (equal to the volume of the i.t. catheter). I.c.v. injection volumes were $1 \mu \mathrm{l}$. Microinjections into specific supraspinal sites were carried out over a period of $90 \mathrm{sec}$, and the injection cannulae were left in place an additional $30 \mathrm{sec}$ after injection.

\section{Results}

\section{Intrathecal DAMGO}

To determine if i.t. opioids modulate nociception via an ascending pathway, the effect of i.t. DAMGO (75 $\mathrm{ng}$ to $7.5 \mathrm{mg}$ ) on the JOR, a reflex which should not be affected directly by i.t. drugs, was determined. Figure 2 illustrates an example of the

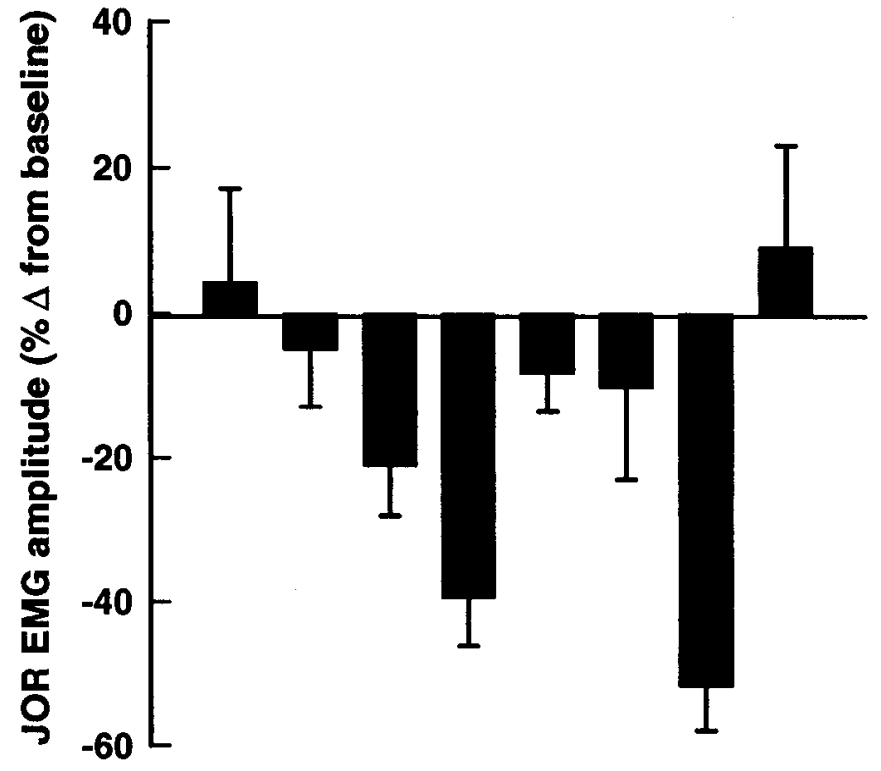

$\ulcorner$ DAMGO $(\mu \mathrm{g})\urcorner$

\section{l.t. veh $.075 .75 \quad 7.57 .5$ veh lido lido i.c.v. veh $\mathrm{nlx}$ nlx $\mathrm{nlx}$

$\begin{array}{llllllllll}\text { n } & & 5 & 5 & 6 & 14 & 14 & 5 & 14 & 6\end{array}$

Figure 3. The effect of i.t. DAMGO, i.t. lidocaine (lido), or i.t. vehicle (veh) with or without i.c.v. naloxone ( $n l x)$ on the amplitude of the JOR. The legend beneath the graph indicates the i.t. treatment, the i.c.v. treatment, and the number of animals in each experimental group. DAMGO (i.t.) dose dependently attenuated the JOR. I.t. lidocaine also showed significant suppression of the JOR. The groups receiving lidocaine or the highest dose of DAMGO (without naloxone) were not significantly different from each other $(p>0.05)$ but were significantly different from the groups receiving i.t. vehicle/i.c.v. vehicle, i.t. vehicle/i.c.v. naloxone, or i.t. DAMGO/i.c.v. naloxone $(p<0.05)$; these last three group were not significantly different from each other $(p>0.05)$. In this and subsequent figures error bars indicate SEM.

JOR EMG waveform before (Fig. $2 A$ ) and $15 \mathrm{~min}$ after (Fig. $2 B$ ) the administration of i.t. DAMGO $(7.5 \mu \mathrm{g})$. DAMGO produced a dose-dependent suppression of the JOR (Fig. 3). I.t. vehicle had no significant effect on the JOR.

To control for systemic absorption of i.t. DAMGO, the highest dose of DAMGO $(7.5 \mu \mathrm{g})$ was also administered intravenously in a different group of animals $(n=4)$. Fifteen minutes after receiving this treatment the JOR amplitude recorded from this group was not significantly different from baseline $(-3 \pm 10 \%$, mean $\pm \mathrm{SEM}, p>0.05$ ).

To determine if attenuation of the JOR by i.t. DAMGO is dependent on supraspinal opioids, i.c.v. naloxone $(2 \mu \mathrm{g})$ was administered $1 \mathrm{~min}$ before i.t. DAMGO (7.5 $\mu \mathrm{g})$, and the JOR was recorded $15 \mathrm{~min}$ later. In the presence of naloxone (i.c.v.), DAMGO (i.t.) failed to significantly suppress the JOR ( $p<$ 0.05). To determine if i.c.v. naloxone might exert an independent (i.e., hyperalgesic) effect, naloxone was administered i.c.v. with i.t. vehicle. This treatment did not significantly affect JOR amplitude (Fig. 3) $(p>0.05)$.

\section{Intrathecal lidocaine}

Opioid-mediated antinociception at the supraspinal site could be produced either by an excitatory action or by disinhibition. If 


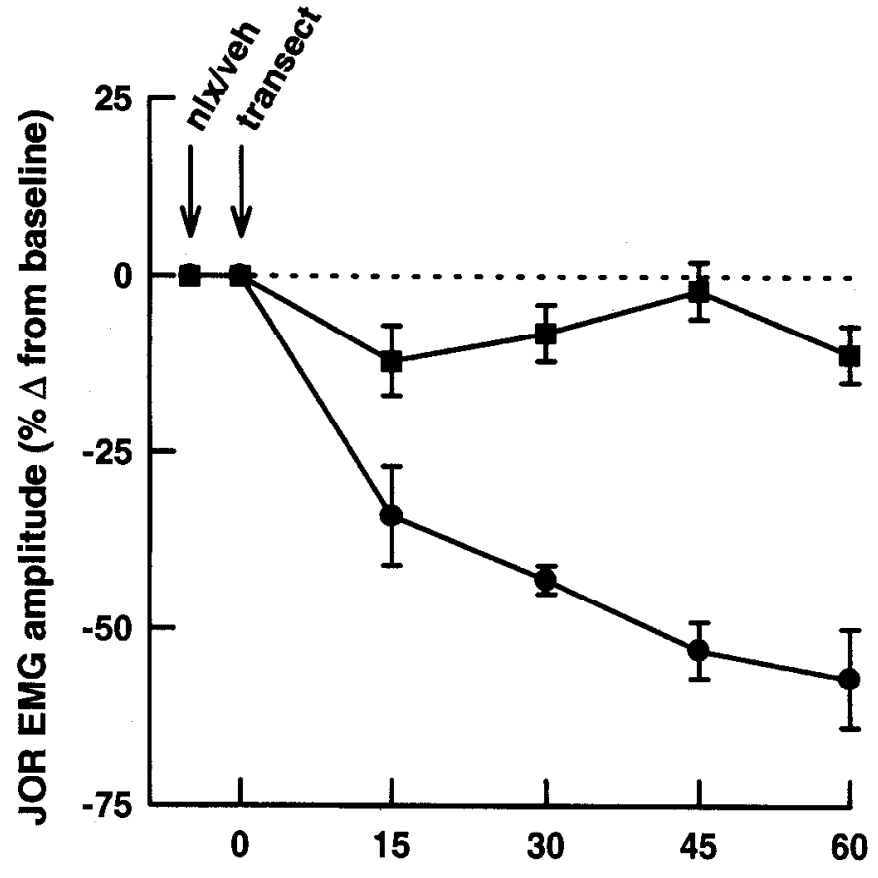

Time post transection (min)

Figure 4. The effect of spinal transection on the JOR with or without i.c.v. naloxone. Spinal transection (transect) in the presence of i.c.v. vehicle (veh) (circles, $n=4$ ) suppressed the JOR, but spinal transection in the presence of i.c.v. naloxone $(n l x)$ (squares, $n=4$ ) remained near baseline levels, a significant difference $(p<0.05)$.

disinhibition is the mechanism, the ascending pathway must be tonically active and the suppression of this tonic activity by a spinally administered local anesthetic should mimic the ability of i.t. DAMGO to suppress the JOR. Therefore, lidocaine (0.6 mg) was administered i.t. Fifteen minutes after i.t. administration, lidocaine suppressed the mean JOR amplitude 52\% below baseline (Fig. 3). Suppression of the JOR by i.t. lidocaine or i.t. DAMGO was not significantly different $(p>0.05)$. These results suggest that the ascending pathway is tonically active and must be suppressed in order to attenuate the JOR.

Since lidocaine might enter the general circulation following i.t. administration and act at a site other than the lumbar spinal cord, the same dose of lidocaine was administered subcutaneously at the nape of the neck in a separate group of animals ( $n$ $=6$ ). Fifteen minutes after receiving this treatment the JOR amplitude recorded from this group was not significantly different from baseline $(+4.2 \pm 6.94 \%$, mean $\pm \mathrm{SEM}, p>0.05)$.

To determine if attenuation of the JOR by i.t. lidocaine is mediated by a supraspinal opioidergic mechanism similar to that mediating the action of i.t. DAMGO, i.c.v. naloxone $(2 \mu \mathrm{g})$ was administered $1 \mathrm{~min}$ before i.t. lidocaine $(0.6 \mathrm{mg})$ and the JOR was recorded $15 \mathrm{~min}$ later. Naloxone completely blocked the ability of lidocaine to attenuate the JOR $(p<0.05)$ (Fig. 3), strongly suggesting that i.t. lidocaine, similar to i.t. DAMGO, attenuates the JOR by a supraspinal opioidergic mechanism.

\section{Spinal transection}

If the ascending antinociceptive system is tonically active, as suggested by the effects of i.t. lidocaine, other methods of suppression of activity in ascending pathways should also produce antinociception/inhibition of the JOR that is antagonized by i.c.v.

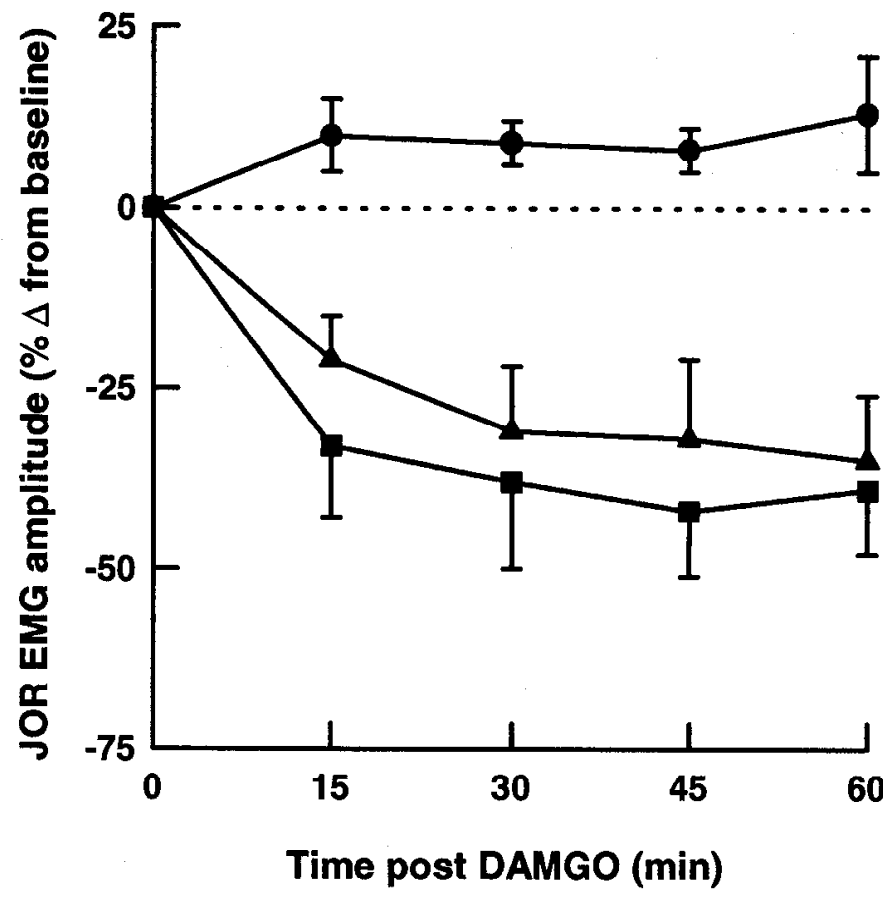

Figure 5. The effect of naloxone methiodide injected i.c.v. or microinjected in RVM or PAG. All groups received i.t. DAMGO. Only the group receiving i.c.v. naloxone methiodide (circles, $n=6$ ) failed to show attenuation of the JOR. The groups receiving naloxone methiodide microinjected into either RVM ( $1 \mu \mathrm{g}$ in $0.5 \mu \mathrm{l} / \mathrm{side}$, squares, $n=6$; sites plotted in Fig. $7 B)$ or PAG $(2 \mu \mathrm{g}$ in $0.5 \mu \mathrm{l}$, triangles, $n=6$; sites plottcd in Fig. 7A) wcre significantly different from the group receiving i.c.v. naloxone methiodide $(p<0.05)$, but were not significantly different from each other $(p>0.05)$.

naloxone. Therefore, we next performed spinal transection at the $\mathrm{T}_{5}-\mathrm{T}_{6}$ level. To determine if a supraspinal opioidergic mechanism is involved, spinal transection was performed in the presence of either naloxone or vehicle administered i.c.v. The following protocol was performed: (1) JOR baseline measurements were recorded in acutely laminectomized animals, (2) i.c.v. naloxone (2 $\mu \mathrm{g}$ ) or vehicle was administered, (3) 5 min later the spinal cord was transected, (4) JOR was measured at $15 \mathrm{~min}$ inter vals for 1 hr. (Although acute spinal transection can lead to hyperresponsiveness of spinal reflexes, in our experiments the JOR did not demonstrate hyperresponsiveness.) The group receiving i.c.v. vehicle showed significant JOR attenuation compared to the group receiving i.c.v. naloxone (Fig. 4) $(p<0.05)$. I.c.v. naloxone almost completely blocked the ability of spinalization to attenuate the JOR suggesting that this attenuation is mediated by a supraspinal opioidergic mechanism similar to that mediating the effects of intrathecally administered lidocaine or DAMGO.

\section{Supraspinal sites}

To locate the supraspinal site at which endogenous opioids contribute to the ascending antinociception produced by i.t. DAMGO, we first evaluated the effect of the injection of naloxone methiodide into the PAG and the RVM, two supraspinal sites that contribute to opioid analgesia in descending antinociceptive systems. Ventrolateral PAG sites were chosen because of previous reports implicating these sites in morphine antinociception (Yeung et al., 1977; Yaksh et al., 1988) (sites plotted in Fig. 8). Naloxone methiodide is a quaternary derivative of naloxone chosen because it spreads more slowly than naloxone (Schroeder 


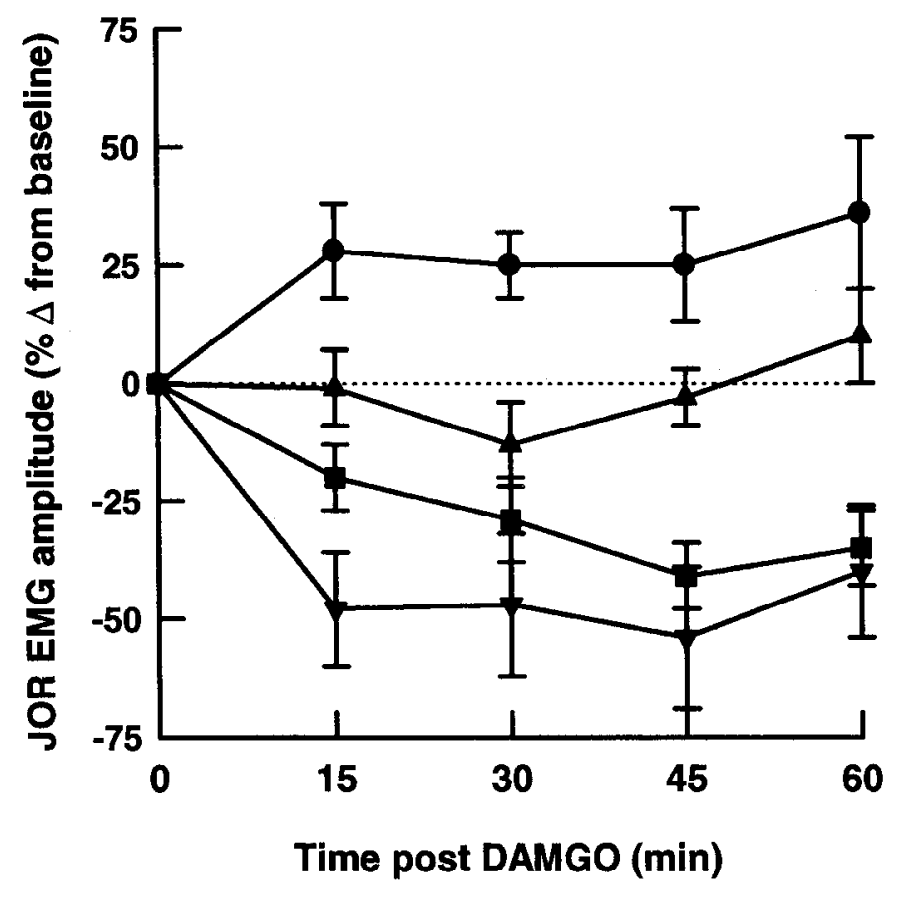

Figure 6. The effect of i.t. DAMGO on the JOR with or without naloxone methiodide microinjected into specific rostral sites. Naloxone methiodide $(1 \mu \mathrm{g}$ in $0.2 \mu \mathrm{l} \mathrm{CSF} /$ side, circles, $n=6$; sites plotted in Fig. $8 A$ ), but not vehicle ( $0.2 \mu \mathrm{l} \mathrm{CSF/side,} \mathrm{squares,} n=6$; sites plotted in Fig. $8 C$ ), microinjected into nucleus accumbens five minutes before i.t. DAMGO prevented suppression of the JOR. Naloxone methiodide ( $1 \mu \mathrm{g}$ in $0.2 \mu \mathrm{l} \mathrm{CSF} /$ side, upward triangles, $n=5$; sites plotted in Fig. $8 D$ ) administered alone to nucleus accumbens had no effect on the JOR. Off-site injections of naloxone methiodide $(1 \mu \mathrm{g}$ in $0.2 \mu \mathrm{l} \mathrm{CSF} / \mathrm{side}$, downward triangles, $n=5$; sites plotted in Fig. $8 B$ ) failed to prevent suppression of the JOR by i.t. DAMGO.

et al., 1991). I.t. DAMGO significantly attenuated the JOR in the groups of rats receiving naloxone methiodide microinjected into RVM or PAG as compared to the group receiving i.c.v. naloxone methiodide (Fig. 5). Thus, the opioidergic mechanisms in RVM or PAG do not appear to be required in order to observe the antinociceptive effect of i.t. DAMGO. Since opioids microinjectcd into a number of supraspinal sites have been shown to be antinociceptive (Yaksh et al., 1976), we tested the ability of bilateral microinjections of naloxone methiodide into several of these sites to antagonize DAMGO (i.t.) suppression of the JOR. Microinjection of naloxone methiodide, but not vehicle, into nucleus accumbens not only blocked suppression of the JOR by i.t. administration of DAMGO $(7.5 \mu \mathrm{g})$, but showed significant overshoot suggesting a hyperalgesic state $(p<0.05)$ (Fig. 6). The specificity of the nucleus accumbens as a site at which opioid-dependent antinociception is evoked by i.t. DAMGO was further confirmed by the observation that microinjection of naloxone methiodide into sites surrounding nucleus accumbens failed to antagonize suppression of the JOR by i.t. DAMGO (see Figs. 6, 9). The groups receiving i.t. DAMGO and either "off-site" injection of naloxone or "on-site" injection of CSF were not significantly different from each other $(p>0.05)$, but were significantly different from either of the groups receiving "on-site" injection of naloxone $(p<0.05)$. Finally, preliminary findings indicate that naloxone methiodide had no effect when microinjected bilaterally into the habenula, or amygdala (data not shown).

To confirm that nucleus accumbens is also the site of the

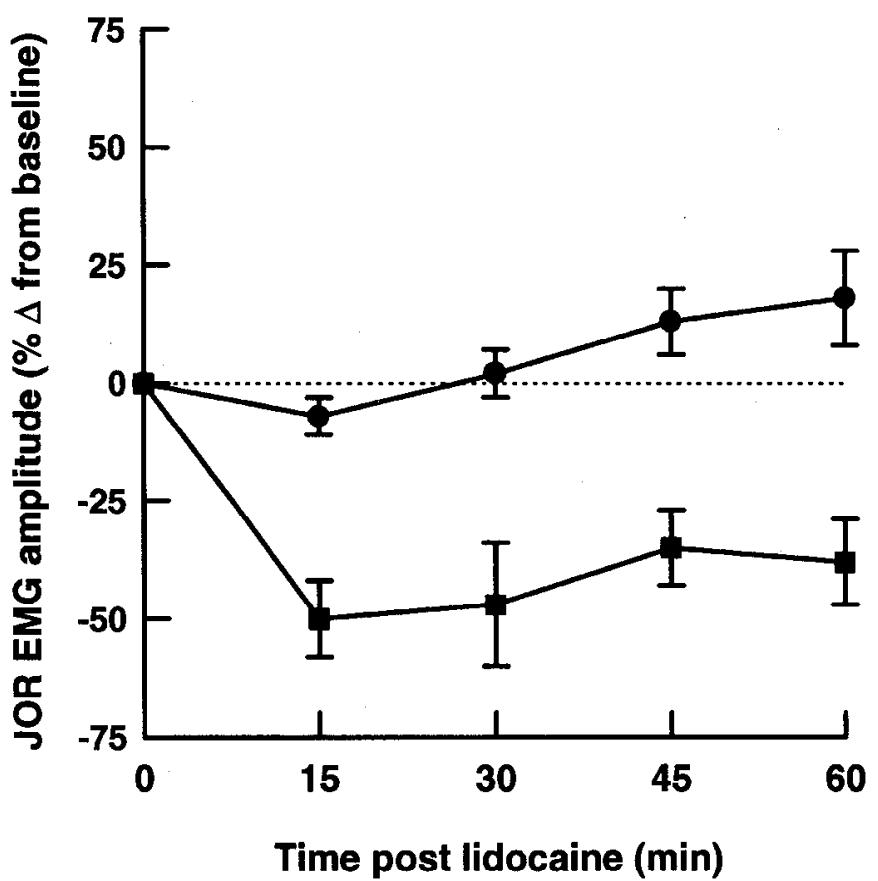

Figure 7. The effect of i.t. lidocaine on the JOR with naloxone methiodide microinjected into specific basal forebrain sites. Naloxone methiodide $(1 \mu \mathrm{g}$ in $0.2 \mu \mathrm{l} \mathrm{CSF} /$ side, circles, $n=6$; sites plotted in Fig. $9 A)$ microinjected into nucleus accumbens five minutes before i.t. lidocaine prevented suppression of the JOR. Off-site injections of naloxone methiodide ( $1 \mu \mathrm{g}$ in $0.2 \mu \mathrm{l} \mathrm{CSF} /$ side, squares, $n=5$; sites plotted in Fig. $9 B$ ) failed to prevent suppression of the JOR by i.t. lidocaine.

opioid link mediating suppression of the JOR by i.t. lidocaine, we tested the ability of bilateral microinjections of naloxone methiodide to block suppression of the JOR by i.t. lidocaine. Naloxone methiodide microinjected into nucleus accumbens, but not into surrounding sites, blocked suppression of the JOR by i.t. lidocaine $(p<0.05)$ (Fig. 7; see Fig. 9). These findings confirm that an opioid link in nucleus accumbens mediates suppression of the JOR by either i.t. lidocaine or i.t. DAMGO, and also confirm that the ascending pathway is tonically active and must be suppressed in order to disinhibit this opioid link in nucleus accumbens.

\section{Discussion}

Spinally evoked antinociception mediated by supraspinal opioids

In this study we demonstrate in the lightly anesthetized rat the existence of a pathway that ascends from the spinal cord to a supraspinal site that produces antinociception mediated by supraspinal opioidergic mechanisms by showing that (1) the JOR is suppressed by DAMGO or lidocaine administered intrathecally at the lumbar level of the spinal cord, or by spinal transection, and (2) that supraspinally administered naloxone (i.e., i.c.v. or in nucleus accumbens) antagonizes this effect. The observation that i.t. lidocaine or spinal transection mimic i.t. DAMGO in suppressing the JOR suggests that the ascending pathway is tonically active. We propose that inhibition of activity in this ascending pathway by spinal analgesic agents (i.e., opioids or local anesthetics) disinhibits supraspinal opioid-mediated antinociception. This supraspinal antinociceptive mechanism appears to have a global effect on nociceptive reflexes as it has been detected at the site of lumbar drug administration (Holmes and 
A

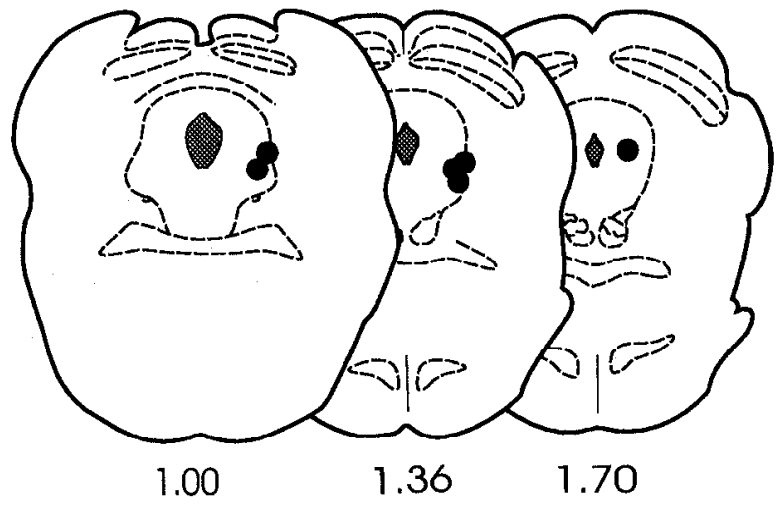

B

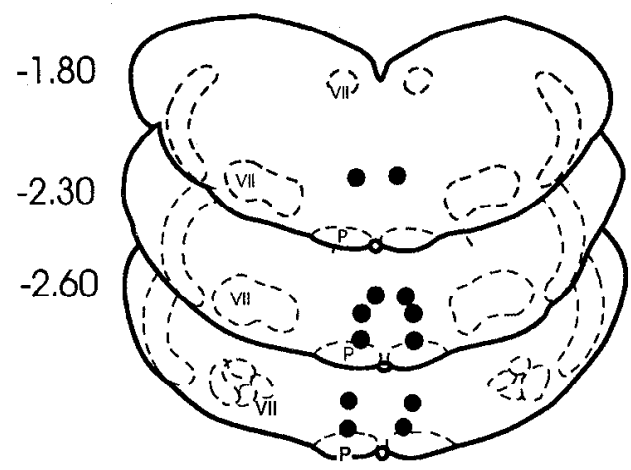

Figure 8. A, Locations of naloxone methiodide injections plotted on coronal sections of PAG. In this and following figures numbers refer to distance (millimeters) caudal (negative numbers) or rostral to the interaural line of coronal sections adapted from the atlas of Paxinos and Watson (Paxinos and Watson, 1986). B, Locations of naloxone methiodide plotted on coronal sections of RVM adapted from the atlas of Paxinos and Watson (Paxinos and Watson, 1986). VII, Facial nucleus; $P$, pyramidal tract.

Fujimoto, 1992; Miaskowski and Levine, 1992) as well as a trigeminal nociceptive reflex (i.e., the JOR). Also, the reported observation that morphine, administered intrathecally to the lumbar spinal cord, is effective in the treatment of head and neck cancer pain (Andersen et al., 1991) suggests that the ascending pathway may be relevant to the treatment of pain. In addition, a number of studies have reported that spinally administered local anesthetics potentiate the antinociceptive effects of spinal morphine (Akerman et al., 1988; Penning and Yaksh, 1990; Maves and Gebhart, 1992). Given our current findings, it is possible that this potentiation is mediated by suppression of activity in the ascending pathway which disinhibits a supraspinal opioidergic mechanism. At present, nothing is known of the physiological conditions under which antinociception mediated by inhibition of the ascending pathway might occur. Since endogenous opioids are released in the spinal cord under various conditions (Watkins et al., 1982; Yaksh et al., 1983; Chung et al., 1984; Cesselin et al., 1985; Le Bars et al., 1987; Bourgoin et al., 1990; Taylor et al., 1990), and since we have shown that intrathecal opioids produce antinociception via the ascending pathway, we suggest that endogenously released spinal opioids might act on the ascending pathway in a similar manner.

Site of action of supraspinal naloxone

In this study we demonstrate that microinjection of naloxone methiodide into the nucleus accumbens, but not into several oth-
A

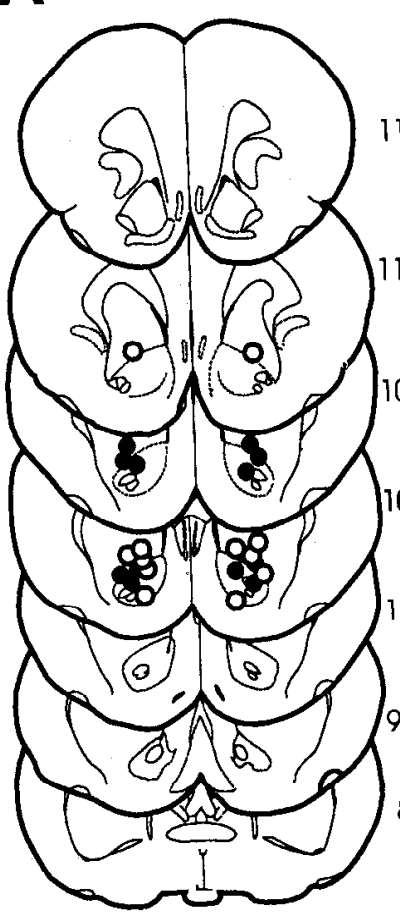

8
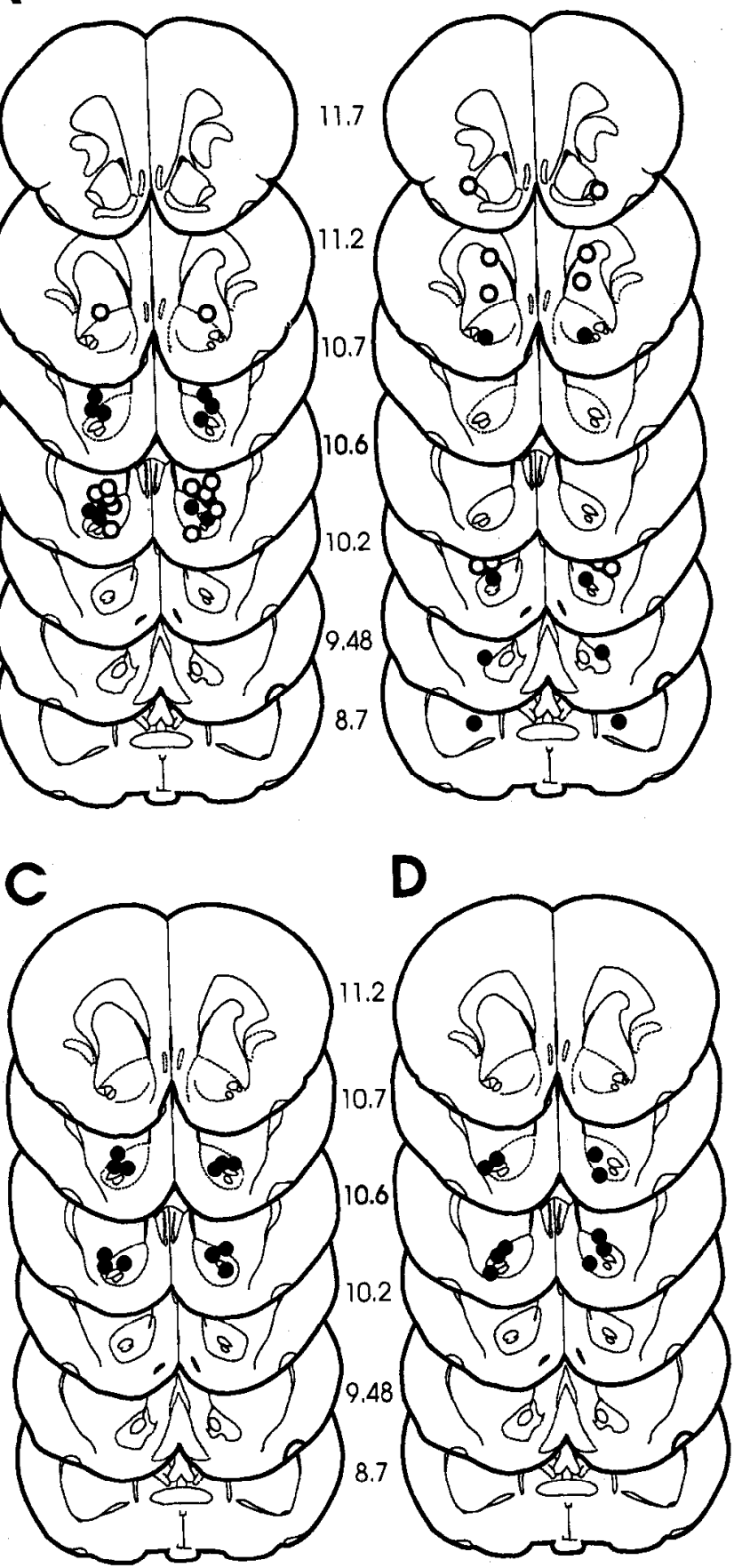

Figure 9. Locations of injection sites in nucleus accumbens. Solid circles (cxcept $D$ ) indicate that the spinal drug was D $\Lambda$ MGO, open circles indicate that the spinal drug was lidocaine. $A$, "on-site" (i.e., in nucleus accumbens) locations of naloxone methiodide injections; $B$, "off-site" (i.e., adjacent to nucleus accumbens) locations of maloxone methiodide injections; $C$, "on-site" locations of CSF injections; and $D$, "on-site" locations of naloxone methiodide injected as a single agent.

er supraspinal sites (i.e., RVM, PAG, or sites adjacent to nucleus accumbens), blocks the suppression of the JOR by either i.t. DAMGO or i.t. lidocaine. These results suggest that nucleus accumbens contains an opioidergic mechanism important in mediating the antinociceptive effect of i.t. DAMGO as well as i.t. lidocaine, and further suggest that this opioid mechanism is ac- 
tivated by disinhibition (i.e., suppression of tonic activity in the ascending pathway). In support of a role for the nucleus accumbens in processing nociceptive information, several investigators have reported that microinjection of morphine into nucleus accumbens produces antinociception (Dill and Costa, 1977; Jin et al., 1986; Yu and Han, 1990). Furthermore, nucleus accumbens contains opioid receptors (Atweh and Kuhar, 1977; Stein et al., 1992), and is immunoreactive for both met-enkephalin and $\beta$-endorphin (Hong et al., 1977; Ma and Han, 1991; Ma et al., $1992 b)$. Of note, Han and colleagues have proposed the existence of a "mesolimbic loop of analgesia" in which the opioid circuitry in the nucleus accumbens plays an important role (Han and Xuan, 1986; Xuan et al., 1986; Yu and Han, 1990; Ma and Han, 1991; Ma et al., 1992a,b). Spinal neurons which carry nociceptive information have been shown to project directly to nucleus accumbens and other limbic structures (Burstein et al., 1987; Burstein and Giesler, 1989; Cliffer et al., 1991).

\section{Diffuse noxious inhibitory controls (DNIC)}

Antinociception produced via the ascending pathway appears to resemble DNIC in that an event remote from the site of application of a noxious stimulus is capable of raising the threshold of response to that stimulus (see Le Bars et al., 1992, for review of DNIC). The antinociception produced by the ascending pathway and that produced by DNIC are, however, likely to result from different mechanisms since DNIC is mediated by excitatory activity in ascending pathways (Le Bars and Villanueva, 1988; Villanueva et al., 1988), whereas we demonstrate that inhibition of tonic activity in ascending pathways evokes antinociception.

\section{Relevance to awake, pain-free state}

Since, in our experiments, we used lightly anesthetized animals in an acute preparation, it is possible that animals in this state could be exhibiting a phenomenon not present in animals in an awake, pain-free state. However, a strong argument against this is that antinociception produced by spinally administered opioids is also antagonized by supraspinal opioid antagonists in awake, pain-free animals (Holmes and Fujimoto, 1992; Miaskowski and Levine, 1992). Nevertheless, it is important that our findings be confirmed in other experimental paradigms which avoid the use of anesthesia and procedures which stimulate nociceptors.

In summary, we demonstrate that either a spinally administered opioid (DAMGO) or a spinally administered local anesthetic (lidocaine) attenuates the trigeminal JOR and that in either case this attenuation is blocked by the administration of naloxone methiodide into the nucleus accumbens. Spinal transection also suppress the JOR in a manner sensitive to supraspinal naloxone. These observations support the suggestion that suppression of tonic activity in an ascending pathway disinhibits a supraspinal antinociceptive circuit with an opioid link in nucleus accumbens (Fig. 1A). This inhibition of supraspinal opioid-dependent antinociception by ascending tonic activity implies that the net effect of spinal input into the limbic system is to facilitate nociceptive sensitivity. This facilitation may be suppressed by events that evoke the release of spinal endogenous opioids.

\section{References}

Akerman B, Arwestrom E, Post C (1988) Local anesthetics potentiate spinal morphine antinociception. Anesth Analg 67:943-948.

Andersen PE, Cohen JI, Everts EC, Bedder MD, Burchiel KJ (1991) Intrathecal narcotics for relief of pain from head and neck cancer. Arch Otolaryngol Head Neck Surg 117:1277-1280.
Atweh SF, Kuhar MJ (1977) Autoradiographic localization of opiate receptors in rat brain. III. The telencephalon. Brain Res 134:393-405.

Basbaum AI, Fields HL (1978) Endogenous pain control mechanisms: review and hypothesis. Ann Neurol 4:451-462.

Basbaum AI, Fields HL (1984) Endogenous pain control system: brainstem spinal pathways and endorphin circuitry. Annu Rev Neurosci 7:309-338.

Bourgoin S, Le Bars D, Clot AM, Hamon M, Cesselin F (1990) Subcutaneous formalin induces a segmental release of Met-enkephalinlike material from the rat spinal cord. Pain 41:323-329.

Burstein R, Giesler GJ Jr (1989) Retrograde labeling of neurons in spinal cord that project directly to nucleus accumbens or the septal nuclei in the rat. Brain Res 497:149-154.

Burstein R, Cliffer KD, Giesler GJ Jr (1987) Direct somatosensory projections from the spinal cord to the hypothalamus and telencephalon. J Neurosci 7:4159-4164.

Cesselin F, Le Bars D, Bourgoin S, Artaud F, Gozlan H, Clot AM, Besson JM, Hamon M (1985) Spontaneous and evoked release of methionine-enkephalin-like material from the rat spinal cord in vivo. Brain Res 339:305-313.

Chung JM, Fang ZR, Hori Y, Lee KH, Willis WD (1984) Prolonged inhibition of primate spinothalamic tract cells by peripheral nerve stimulation. Pain 19:259-275.

Cliffer KD, Burstein R, Giesler GJ Jr (1991) Distributions of spinothalamic, spinohypothalamic, and spinotelencephalic fibers revealed by anterograde transport of PHA-L in rats. J Neurosci 11:852-868.

Dill RE, Costa E (1977) Behavioural dissociation of the enkephalinergic systems of nucleus accumbens and nucleus caudatus. Neuropharmacology 16:323-326.

Fields HL, Basbaum AI (1994) Central nervous system mechanisms of pain modulation. In: Textbook of pain (Wall PD, Melzack R, eds), pp 243-257. Edinburgh: Churchill Livingstone.

Fisher RA (1949) The design of experiments. Edinburgh: Oliver and Boyd.

Gear RW, Levine JD (1994) A tonically ascending pathway suppresses antinociception mediated by supraspinal endogenous opioids. Soc Neurosci Abstr 20:1.15.

Han JS, Xuan YT (1986) A mesolimbic neuronal loop of analgesia. I. Activation by morphine of a serotonergic pathway from periaqueductal gray to nucleus accumbens. Int J Neurosci 29:109-117.

Holmes BB, Fujimoto JM (1992) Naloxone and norbinaltorphimine administered intracerebroventricularly antagonize spinal morphineinduced antinociception in mice through the antianalgesic action of spinal dynorphin A (1-17). J Pharmacol Exp Ther 261:146-153.

Hong JS, Yang H-YT, Fratta W, Costa E (1977) Determination of methionine enkephalin in discrete regions of rat brain. Brain Res 134: 383-386.

Jin WQ, Zhou ZF, Han JS (1986) Electroacupuncture and morphine analgesia potentiated by bestatin and thiorphan administered to the nucleus accumbens of the rabbit. Brain Res 380:317-324.

Le Bars D, Villanueva L (1988) Electrophysiological evidence for the activation of descending inhibitory controls by nociceptive afferent pathways. Prog Brain Res 77:275-299.

Le Bars D, Bourgoin S, Clot AM, Hamon M, Cesselin F (1987) Noxious mechanical stimuli increase the release of Met-enkephalin-like material heterosegmentally in the rat spinal cord. Brain Res 402:188192.

Le Bars D, Villanueva L, Bouhassira D, Willer JC (1992) Diffuse noxious inhibitory controls (DNIC) in animals and in man. Patol Fiziol Eksp Ter 55-65.

Leviel V, Gobert A, Guibert B (1989) Direct observation of dopamine compartmentation in striatal nerve terminal by 'in vivo' measurement of the specific activity of released dopamine. Brain Res 499:205213.

Lux F, Welch SP, Brase DA, Dewey WL (1988) Interaction of morphine with intrathecally administered calcium and calcium antagonists: evidence for supraspinal endogenous opioid mediation of intrathecal calcium-induced antinociception in mice. J Pharmacol Exp Ther 246: 500-507.

Ma QP, Han JS (1991) Neurochemical studies on the mesolimbic circuitry of antinociception. Brain Res 566:95-102.

Ma QP, Shi YS, Han JS (1992a) Further studies on interactions between periaqueductal gray, nucleus accumbens and habenula in antinociception. Brain Res 583:292-295.

Ma QP, Shi YS, Han JS (1992b) Naloxone blocks opioid peptide re- 
lease in N. accumbens and amygdala elicited by morphine injected into periaqueductal gray. Brain Res Bull 28:351-354.

Maves TJ, Gebhart GF (1992) Antinociceptive synergy between intrathecal morphine and lidocaine during visceral and somatic nociception in the rat. Anesthesiology 76:91-99.

Miaskowski C, Levine JD (1992) Inhibition of spinal opioid analgesia by supraspinal administration of selective opioid antagonists. Brain Res 596:41-45.

Paxinos G, Watson C (1986) The rat brain in stereotaxic coordinates. New York: Academic.

Penning JP, Yaksh TL (1990) The analgesic interaction between intrathecal morphine, lidocaine and bupivacaine in the rat. Can J Anaesth 37:S48.

Schroeder RL, Weinger MB, Vakassian L, Koob GF (1991) Methylnaloxonium diffuses out of the rat brain more slowly than naloxone after direct intracerebral injection. Neurosci Lett 121:173-177.

Stein EA, Hiller JM, Simon EJ (1992) Effects of stress on opioid receptor binding in the rat central nervous system. Neuroscience 51: 683-690.

Taylor JS, Pettit JS, Harris PJ, Ford TW, Clarke RW (1990) Noxious stimulation of the toes evokes long-lasting, naloxone-reversible suppression of the sural-gastrocnemius reflex in the rabbit. Brain Res $531: 263-268$

Toda K, Iriki A, Ichioka M (1981) Selective stimulation of intrapulpal nerve of rat lower incisor using a bipolar electrode method. Physiol Behav 26:307-311.

Villanueva L, Bouhassira D, Bing Z, Le Bars D (1988) Convergence of heterotopic nociceptive information onto subnucleus reticularis dorsalis neurons in the rat medulla. J Neurophysiol 60:980-1009.

Watkins LR, Cobelli DA, Mayer DJ (1982) Classical conditioning of front paw and hind paw footshock induced analgesia (FSIA): naloxone reversibility and descending pathways. Brain Res 243:119132.

Welch SP, Stevens DL, Dewey WL (1992) A proposed mechanism of action for the antinociceptive effect of intrathecally administered calcium in the mouse. J Pharmacol Exp Ther 260:117-127.

Xuan YT, Shi YS, Zhou ZF, Han JS (1986) Studies on the mesolimbic loop of antinociception. II. A serotonin-enkephalin interaction in the nucleus accumbens. Neuroscience 19:403-409.

Yaksh TL, Rudy TA (1976) Chronic catheterization of the spinal subarachnoid space. Physiol Behav 17:1031-1036.

Yaksh TL, Terenius L, Nyberg F, Jhamandas K, Wang JY (1983) Studies on the release by somatic stimulation from rat and cat spinal cord of active materials which displace dihydromorphine in an opiatebinding assay. Brain Res 268:119-128.

Yaksh TL, Al-Rhodhan NRF, Jensen TS (1988) Sites of action of opiates in production of analgesia. Prog Brain Res 77:377-394.

Yeung JC, Yaksh TL, Rudy TA (1977) Concurrent mapping of brain sites for sensitivity to the direct application of morphine and focal electrical stimulation in the production of antinociception in the rat. Pain 4:23-40.

Yu LC, Han JS (1990) The neural pathway from nucleus accumbens to amygdala in morphine analgesia of the rabbit. Sheng $\mathrm{Li} \mathrm{Hsuch}$ Pao 42:277-283. 